Article

\title{
Two Cycloartenol Synthases for Phytosterol Biosynthesis in Polygala tenuifolia Willd
}

\author{
Mei Lan Jin, Woo Moon Lee and Ok Tae Kim* \\ Department of Herbal Crop Research, National Institute of Horticultural and Herbal Science, \\ Rural Development Administration, Eumseong 27709, Korea; milan80623@korea.kr (M.L.J.); \\ wmlee65@korea.kr (W.M.L.) \\ * Correspondence: kimot99@hanmail.net; Tel.: +82-63-238-6123; Fax: +82-63-238-6105
}

Received: 22 October 2017; Accepted: 11 November 2017; Published: 15 November 2017

\begin{abstract}
Oxidosqualene cyclases (OSCs) are enzymes that play a key role in control of the biosynthesis of phytosterols and triterpene saponins. In order to uncover OSC genes from Polygala tenuifolia seedlings induced by methyl jasmonate (MeJA), RNA-sequencing analysis was performed using the Illumina sequencing platform. A total of 148,488,632 high-quality reads from two samples (control and the MeJA treated) were generated. We screened genes related to phytosterol and triterpene saponin biosynthesis and analyzed the transcriptional changes of differentially expressed unigene (DEUG) values calculated by fragments per kilobase million (FPKM). In our datasets, two full-length cDNAs of putative OSC genes, PtCAS1, and PtCAS2, were found, in addition to the PtBS ( $\beta$-amyrin synthase) gene reported in our previous studies and the two cycloartenol synthase genes of P. tenuifolia. All genes were isolated and characterized in yeast cells. The functional expression of the two PtCAS genes in yeast cells showed that the genes all produce a cycloartenol as the sole product. When qRT-PCR analysis from different tissues was performed, the expressions of PtCAS1 and PtCAS2 were highest in flowers and roots, respectively. After MeJA treatment, the transcripts of PtCAS1 and PtCAS2 genes increased by 1.5- and 2-fold, respectively. Given these results, we discuss the potential roles of the two PtCAS genes in relation to triterpenoid biosynthesis.
\end{abstract}

Keywords: cycloartenol synthase; oxidosqualene cyclase; phytosterols; triterpenoids; Polygala tenuifolia

\section{Introduction}

The cyclization of 2,3-oxidosqualene, which is catalyzed by several oxidosqualene cyclases (OSCs) results in the formation of various triterpenoids. These processes are completed through the protonation of epoxide, rearrangement of carbocation, and termination by deprotonation [1]. A protosteryl cation can convert 2,3-oxidosqualene to lanosterol, cycloartenol, and curcurbitadinol of chair-boat-chair type series reactions; and a dammarenyl cation can convert lupeol, $\beta$-amyrin, taraxerol, and other triterpenoids of chair-chair-chair type series reactions [2]. Cycloartenol synthase (CAS, EC 5.4.99.8) catalyzes the cyclization of 2,3-oxidosqualene and finally produces a cycloartenol. Phytosterols, which are biosynthesized through oxidation and hydroxylation, are an essential element for forming plasma membranes. The most common sterols that accumulate in plants are $\beta$-sitosterol, stigmasterol, and campesterol (Figure 1). Recently, it has been reported that the CAS protein plays a vital role in sterol biosynthesis, which is essential for plant cell viability [3]. Consistent with this, the cas1 mutant lines of Arabidopsis exhibit lower levels of sterols than the control. Only one CAS (cycloartenol synthase) gene exists in the Arabidopsis genome [4], whereas two CAS genes, BPX and BPX2, from the Butula platyphylla were cloned and characterized in yeast cells [5]. Thus far, any advanced studies on the different functions between the $C A S$ genes of a plant have not yet been reported. 


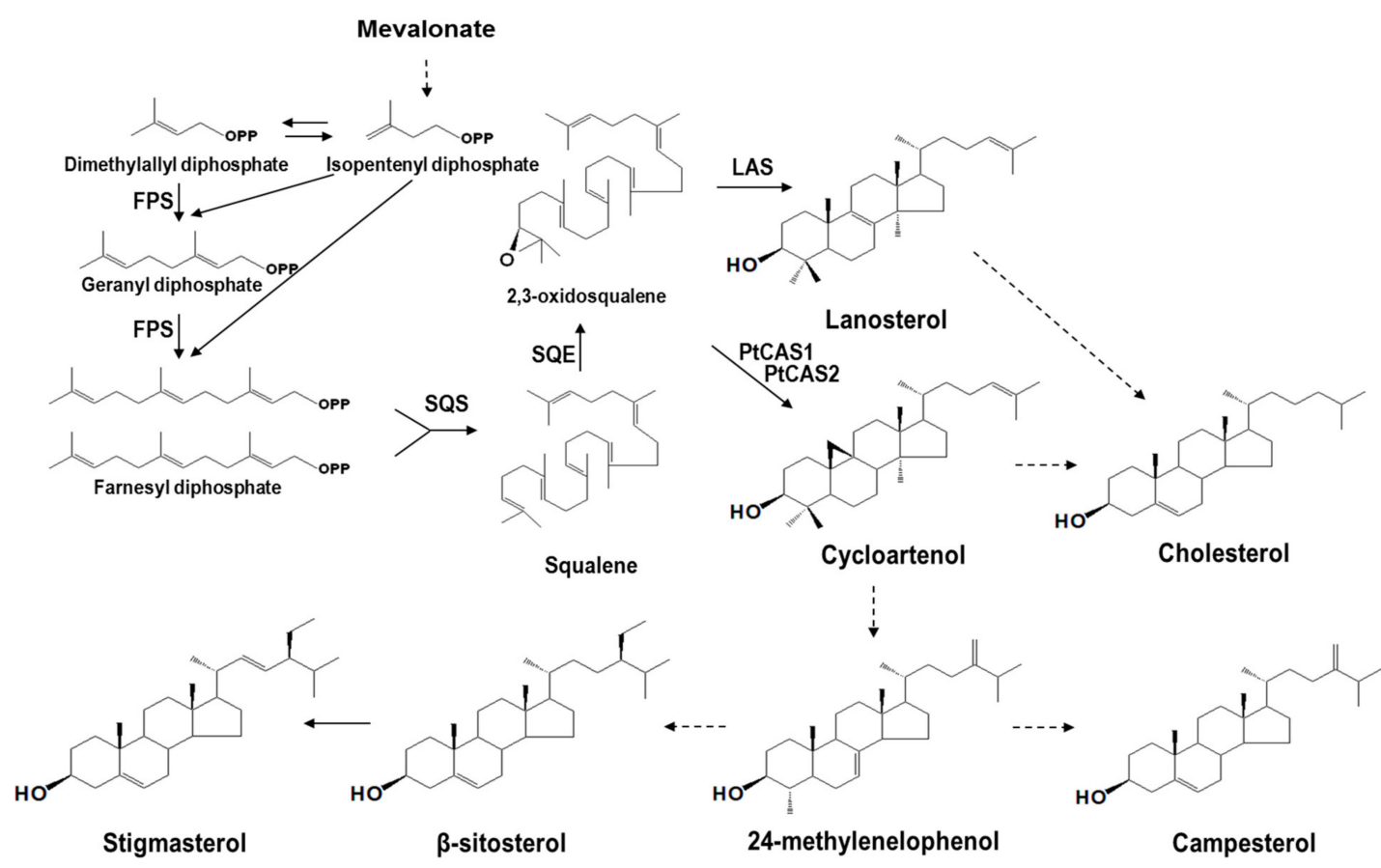

Figure 1. Phytosterol biosynthesis in P. tenuifolia. FPS, farnesyl diphosphate synthase; SQS, squalene synthase; SQE, squalene epoxidase; LAS, lasnosterol synthase. Dashed lines indicate multi-steps.

Polygala tenuifolia, which is a well-known traditional Asian medicine for treating phlegm and for detumescence, is a perennial herb widely distributed in the north of China and Korea. In China, triterpene saponins, which are derived from this herb, were one of the principal components of some medicines, given their chemical and pharmacological properties [6]. P. tenuifolia contains 34 different oleanane-type triterpene saponins, belonging to the groups of onjisaponins and polygalasaponins [7]. However, to date, steroidal saponins as secondary metabolites have not been identified in the plant.

To uncover OSC genes of the $P$. tenuifolia, we employed the Illumina sequencing platform for transcriptomic analysis of 5-week old seedlings induced by methyl jasmonate (MeJA), which is a signaling molecule involved in the regulation of defense mechanisms against fungal pathogens, environmental stress, and injury [8,9]. In particular, MeJA can stimulate not only the production of secondary metabolites such as sesquiterpenoids and triterpenoids but also the high expression of genes associated with pathways involved in cell suspension, hairy root, and adventitious root cultures (e.g., $\beta$-amyrin synthase (bAS), cytochrome P450 (CYP), and glucosyltransferase (UGT)). Recently, Tian et al. [10] reported the results of a transcriptome analysis of wild P. tenuifolia roots. In their study, a total of 70 unigenes of OSCs ( 16 for a CAS and 54 for a bAS) were discovered from the dataset, although the information on the unigenes was not described in detail.

In this study, two cDNA libraries were constructed from 5-week old (24 h) MeJA treated or untreated seedlings, in order to uncover candidate genes related to the triterpenoid pathway by using a high-throughput Illumina deep-sequencing technique. Three genes of approximately $2.3 \mathrm{~kb}$ in size, likely to be full-length cDNAs of OSC, were determined. Two putative CAS genes were isolated from the cDNA pool and characterized by heterologous expression in yeast mutant cells. Further, the expression of the CAS genes in P. tenuifolia was investigated by real-time RT-PCR analysis.

\section{Results}

\subsection{De Novo Transcriptome Assembly and Functional Annotation}

To generate the transcriptome of P. tenuifolia, total RNAs isolated from 5-week old seedlings treated with MeJA were subjected to sequencing on an Illumina platform. A total of $148,488,632$ 
high-quality reads were generated: 71.8 million for the control sample and 76.7 million for the MeJA treated sample (Table 1). De novo assembly of total high-quality reads resulted in the generation of 129,035 unigenes with a GC content of about $45.3 \%$, where the average length of each unigene was $1065 \mathrm{bp}$. A total of 129,035 unigenes were expressed, with the greatest abundance observed with the sample treated with MeJA. Out of the expressed unigenes, a total of 54,931 (42.6\%), 69,060 (53.5\%), and 66,649 (55.5\%) were identified by subjecting them to the NR (NCBI non-redundant protein), TAIR (The Arabidopsis Information Resource) and COG (Clusters of Orthologous Groups) databases, respectively. To understand the characteristics of the functional distribution of genes in P. tenuifolia, we used eggNOG annotation [11], which is based on the COG database. A total of 55,242 unigenes were classified into 25 groups according to their possible functions (Figure 2). Three categories predominated the unigenes: "General function", "Signal transduction", and "Protein turnover" $(16 \%, 7 \%$, and $7 \%$, respectively). In contrast, "Extracellular" and "Nuclear structures" showed the least correlation.

Table 1. Summary of $P$. tenuifolia sequencing and assembly. MeJa = methyl jasmonate.

\begin{tabular}{llc}
\hline Dataset Name & Control & MeJA (24 h) \\
\hline No. raw reads & $71,835,350$ & $76,653,282$ \\
No. clean reads & $66,602,004$ & $71,347,274$ \\
No. of unigenes & 129,035 & \\
Average length of unigenes & $1065 \mathrm{bp}$ & \\
Unigenes expressed in each sample (No.) & 121,987 & 102,869 \\
\hline
\end{tabular}

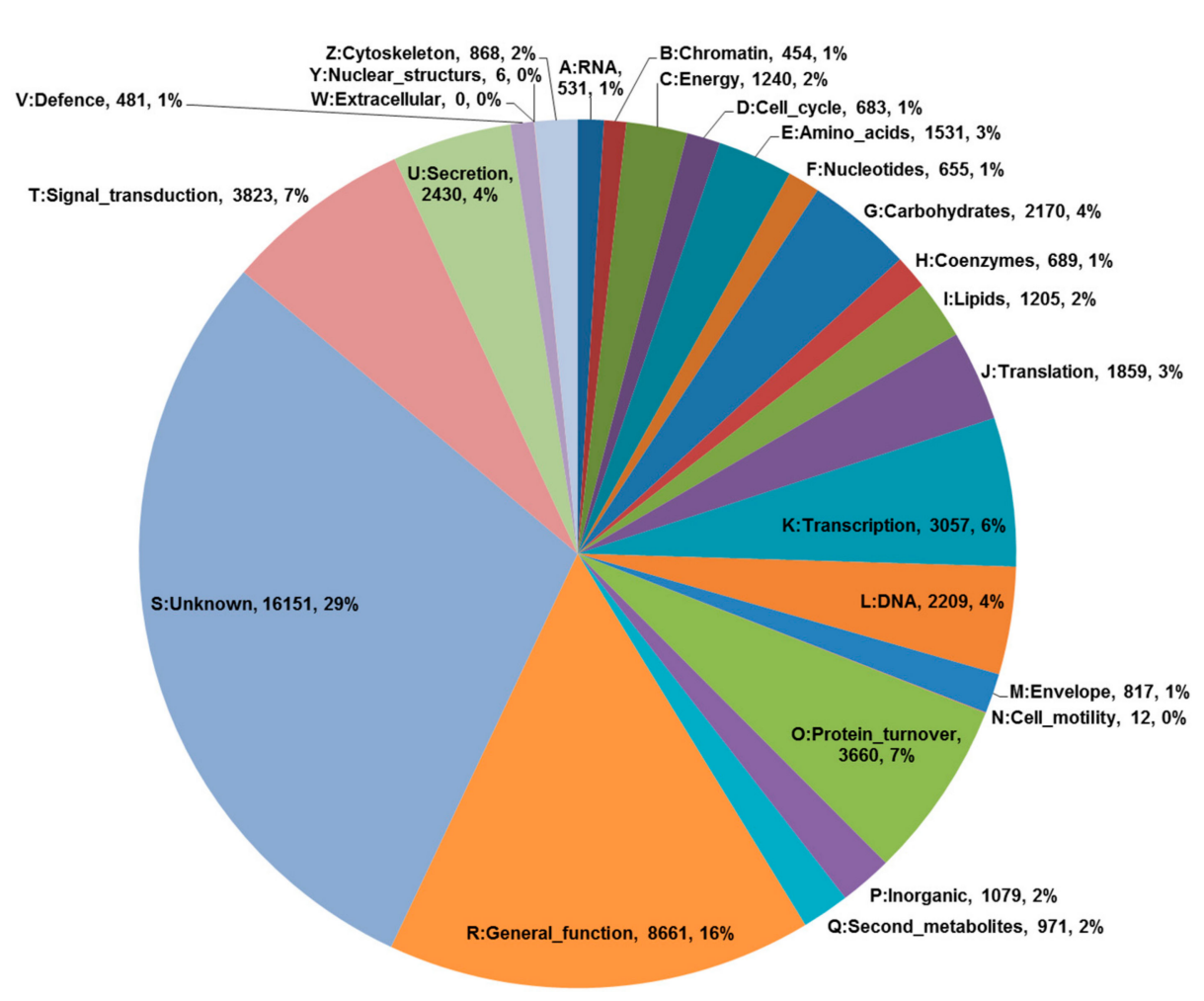

Figure 2. Results of an eggNOG functional category analysis of 55,242 unigenes. All identified proteins were classified into molecular families.

\subsection{Simple Sequence Repeats (SSRs) in the P. tenuifolia Transcriptome}

In this work, a large number of SSRs were identified in the transcriptome data. Based on the motifs, all SSRs were divided into repeats: from di- to hexa-nucleotide repeats (Table 2). Out of the repeat motifs in the $P$. tenuifolia transcriptome, tri-nucleotide repeats were the most abundant 
$(1208,61.9 \%)$, followed by di-nucleotide $(574,29.4 \%)$, tetra-nucleotide $(112,5.7 \%)$, hexa-nucleotide $(31,1.6 \%)$, and penta-nucleotide (25, 1.3\%). These SSR subsets can be used extensively for linkage map development, marker assisted selection, genetic diversity studies, and gene flow and environmental studies [12,13]. In particular, transcript-based molecular markers can be considered an effective tool for classifying functional genetic variations. Nevertheless, SSRs from the P. tenuifolia transcriptome have not been reported until now.

Table 2. Simple sequence repeats (SSR) among different nucleotide types found in the transcriptome of P. tenuifolia.

\begin{tabular}{cccccc}
\hline Different Types of SSRs & Number & Proportion (\%) & Type No. & Total Length (bp) & Average Length (bp) \\
\hline Dinucleotide & 574 & 29.4 & 12 & 102,317 & 15 \\
Trinucleotide & 1208 & 61.9 & 60 & 216,304 & 13 \\
Tetranucleotide & 112 & 5.7 & 72 & 20,302 & 17 \\
Pentanucleotide & 25 & 1.3 & 21 & 4439 & 21 \\
Hexanucleotide & 31 & 1.6 & 31 & 5559 & 25 \\
\hline
\end{tabular}

\subsection{Identification of Differential Gene Expression}

To investigate the differential gene expression in response to MeJA, a total of 125,012 differentially expressed unigenes (DEUGs) were analyzed (Table S1). A dramatic increase of expression of numerous genes was observed in response to MeJA. Among the abundant transcripts (as compared to the control), several well-known transcription factors such as ethylene response factor (ERF), WRKY transcription factor, etc. [9] ranked high. Moreover, we found 57 unigenes related to phytostrol biosynthesis (Table S2).

The unigenes encoding enzymes involved in triterpenoid biosynthesis, including squalene synthase (SQS, EC 2.5.1.21), squalene epoxidase (SQE, EC 1.14.99.7), and oxidosqualene cyclase (OSC, EC 5.4.99.8) were identified (Table 3). SQS, which catalyzes head to tail joining of two units of farnesyl diphosphate to squalene, plays a role in providing the first committed precursor for steroid and triterpene biosynthesis. The transcriptome data of $P$. tenuifolia, as determined by RNA-seq, indicated that the sequences share a $99 \%$ identity with the protein sequences of TBIU1284 of P. tenuifolia SQS (DQ672339), registered on the NCBI database. In the next step of biosynthesis, SQE converts squalene into 2,3-oxidosqualene. In $A$. thaliana, six $S Q E$ genes exist in the genome. However, their physiological functions are still unknown, except for the SQE1 gene of $A$. thaliana, which contributes to root and seed development [14]. In our study, five unigenes for SQE were detected in the transcripotome.

Various members of the OSC gene family can generate numerous triterpenoids by cyclization of 2,3-oxidosqualene, which is the branch point between phytosterol and triterpenoid biosynthesis. bAS leads to the synthesis of a lot of oleanane-type triterpenoids, whereas CAS provides phytosterols with the carbon source as a precursor. In our datasets, eleven unigenes for OSC were determined; and three $b A S$ and eight $C A S$ unigenes were subsequently annotated. Out of those, the sequences of three unigenes for bAS were consistent with those of the PtBS gene (EF107623) reported by Jin et al. [15]. Eight unigenes for CAS appeared to have open reading frames (ORFs) while two unigenes possessed full-length cDNAs that were identified by comparing amino acid sequences. We named the genes PtCAS1 and PtCAS2 genes for CAS in P. tenuifolia. The PtCAS1 and PtCAS2 deduced from amino acid sequences are predicted to encode 762 and 764 amino acids with masses of 86.826 and $86.895 \mathrm{kDa}$, respectively. Both genes contain the DCTAE motif and four QXXXGXW motifs as characteristic of OSCs (Figure 3). The two genes also possessed the MWCHCR motif which CAS genes commonly have, but its function in triterpene cyclization is still unclear. To elucidate the phylogenetic relationship of the two PtCASs to the plant OSCs, we constructed a phylogenetic tree based on the deduced amino acid sequences. As predicted, the two genes grouped clearly with CAS (Figure 4). The two CAS genes in P. tenuifoloa were identified with the transcriptome data by RNA-seq analysis. This finding motivated us to elucidate the relationship between the two genes to phytosterol biosynthesis in P. tenuifolia. 
Table 3. Differential expression of uingenes of SQS (squalene synthase), SQE (squalene epoxidase), and OSC (oxidosqualene cyclase) candidate genes involved in the biosynthesis of phytosterol and triterpenoid backbone of $P$. tenuifolia. LFC $=\log$ fold change; FPKM = fragments per kilobase Million; $\mathrm{CON}=$ control.

\begin{tabular}{|c|c|c|c|c|c|c|c|}
\hline \multirow{2}{*}{ Enzyme } & \multirow{2}{*}{ GeneID } & \multirow{2}{*}{ Description } & \multirow{2}{*}{ Identity (\%) } & \multirow{2}{*}{$e$-Value } & \multirow{2}{*}{ LFC } & \multicolumn{2}{|c|}{ FPKM } \\
\hline & & & & & & CON & MeJA \\
\hline SQS & TBIU012384 & squalene synthase (Polygala tenuifolia) & 99 & 0 & 3.31 & 6.8 & 47.19 \\
\hline \multirow{5}{*}{ SQE } & TBIU001989 & squalene epoxidase (Astragalus membranaceus) & 83 & $2.0 \times 10^{-66}$ & 0.765 & 2.84 & 3.37 \\
\hline & TBIU001990 & squalene monooxygenase (Theobroma cacao) & 88 & $2.0 \times 10^{-83}$ & 13.8 & 0 & 17.77 \\
\hline & TBIU020398 & $\begin{array}{l}\text { PREDICTED: squalene epoxidase 3-like } \\
\text { (Tarenaya hassleriana) }\end{array}$ & 86 & $4.0 \times 10^{-41}$ & -2.08 & 1.6 & 0.26 \\
\hline & TBIU062165 & $\begin{array}{l}\text { Squalene monooxygenase, putative } \\
\text { (Ricinus communis) }\end{array}$ & 88 & $3.0 \times 10^{-14}$ & 1.82 & 1.63 & 4.03 \\
\hline & TBIU068277 & $\begin{array}{l}\text { PREDICTED: squalene monooxygenase-like } \\
\text { (Eucalyptus grandis) }\end{array}$ & 88 & $3.0 \times 10^{-71}$ & -1.02 & 8.19 & 2.83 \\
\hline \multirow{11}{*}{ OSC } & TBIU017624 & $\beta$-amyrin synthase (Polygala tenuifolia) & 100 & 0 & 4.39 & 7.69 & 112.6 \\
\hline & TBIU030037 & cycloartenol synthase1 (Polygala tenuifolia) & 99 & $3.0 \times 10^{-102}$ & 1.03 & 11.05 & 15.74 \\
\hline & TBIU030038 & cycloartenol synthase (Glycine max) & 91 & $5.0 \times 10^{-12}$ & 0.473 & 2.15 & 2.09 \\
\hline & TBIU030039 & cycloartenol synthase (Betula platyphylla) & 81 & $1.0 \times 10^{-71}$ & 0.013 & 6.32 & 4.46 \\
\hline & TBIU030040 & $\begin{array}{l}\text { PREDICTED : cycloartenol synthase } \\
\text { (Ziziphus jujube) }\end{array}$ & 80 & 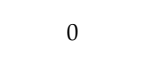 & 0.699 & 2.45 & 2.78 \\
\hline & TBIU030041 & cycloartenol synthase1 (Polygala tenuifolia) & 87 & 0 & 0.932 & 9.48 & 12.63 \\
\hline & TBIU030042 & $\begin{array}{l}\text { PREDICTED : cycloartenol synthase } \\
\text { (Ziziphus jujube) }\end{array}$ & 81 & 0 & 0.043 & 18.15 & 13.06 \\
\hline & TBIU030043 & cycloartenol synthase1 (Polygala tenuifolia) & 87 & 0 & 0.368 & 1.75 & 1.58 \\
\hline & TBIU030044 & cycloartenol synthase1 (Polygala tenuifolia) & 99 & $5.0 \times 10^{-51}$ & 1.12 & 9.07 & 13.84 \\
\hline & TBIU106220 & $\beta$-amyrin synthase (Polygala tenuifolia) & 100 & $1.0 \times 10^{-14}$ & 2.44 & 3.47 & 13.21 \\
\hline & TBIU119757 & $\beta$-amyrin synthase (Polygala tenuifolia) & 99 & $9.0 \times 10^{-56}$ & 2.81 & 3.24 & 15.97 \\
\hline
\end{tabular}

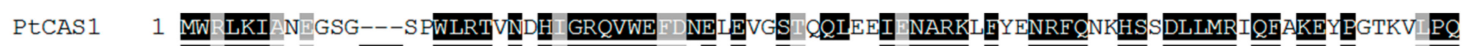

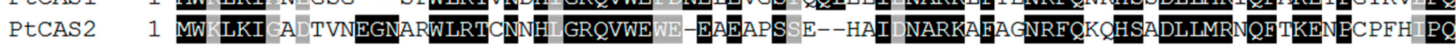

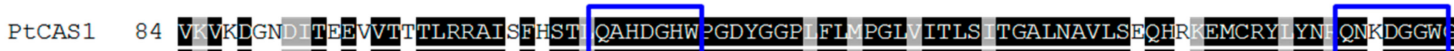
PtCAS2 84 V VDDTEDTEEAVTITLRRAIN YST QAHDGHW PGYGGP FI PGL ITLS TGALNAVLSKQHQ EMCRY YN QNTDGGW PtCAS1 170 LHIEGHST MFG VLNYV LRLLGEG--ANDGEGANER RDW LGHGGATMITSWGKMWLSVLGVYEWSGNNP PPEI

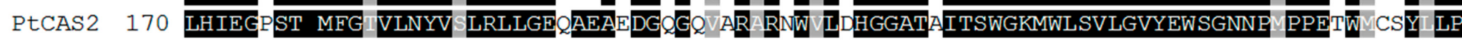
PtCAS1 253 FHPG MWCHCR IVYLPMSYLYGKRFVGPITPTVLSLRKELYNTPYHEINWWD ARSQCAKEDLYYPHPL QD WTSLHK IEPILM PtCAS2 255 FHPG MWCHCR IVYLPMSYLYGKRFVGPITPTVRSLRKELYTI PYHEIDWN ARNTCAKEDLYYPHPL QD WA SLHY

PtCAS1 339 WP K LR KALRTAMEHIHYEDENTRY CIGPVNKALNMLCCW EDPNSEA E LH PRI DYLWIAEDGMKMQGYNGSQLWDTAF PtCAS2 341 WP K LR KALVHVMHHIHYEDENTRY CIGPVNKVLNMLCCW EDPNG ES E LH PRI DYLWIAEDGMKMQGYNGCQLWDTAF PtCAS1 425 AVQAIIS NL EEFEVT RKAHA IKNTQV EDCPGDLHHWYRHISKGAWPFSTADHGWPI:DCTAEC LKAALLLS PSKMGEP PtCAS2 427 AVQAIIS GL EEGAT RKAHE IKDTQV EDCPGDLSFWYRHISKGAWPFSTRDHGWPI: DCTAEC LKA VLLLS LPSEIVGMP PtCAS1 511 LDAKR DAVNVILSLQNPDGGFATYELTRSYPWLELINPAETFGDIVIDYTYVECTSAAIQALVSEKGLYPGYRKEEIERCIEKA PtCAS2 513 LDENR DAVNVILSLQNGDGGFATYELTRSYQWLELINPAETFGDIVIDYPYVECTSAAIQALAIFKKLYPGHR VEIEDCIVKA

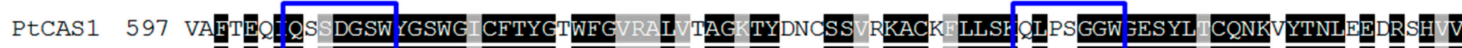
PtCAS2 599 ATEIEK QA DGSW YGSWG CFTYGAWFG KCIAAG TYKDSSSI CKACD LLSK ELAPGGW JESYL CQNKA YTNLKENRPH VV

PtCAS1 683 NTAWA AIFDAGQAQRDEKPLNLAAQY NSQ ENGDFPQEEIMGVFN NCMI YYSAYRNI FPIWALGEYRTRLLAC PLCAS2 685 NTAWA TIVA AGQAERDPT PLHRAARI NSQ ENGDFPQE EIMGVFN NCMI YAAYRNI FP IWALGEYKSK VLHSL
\end{abstract}

Figure 3. Alignment of the deduced amino acid sequences of two PtCASs from P. tenuifolia. Blue, yellow, and red boxes indicate the QXXXGXW motifs, the highly conserved motif among CAS genes, and the DCTAE motif, respectively. 


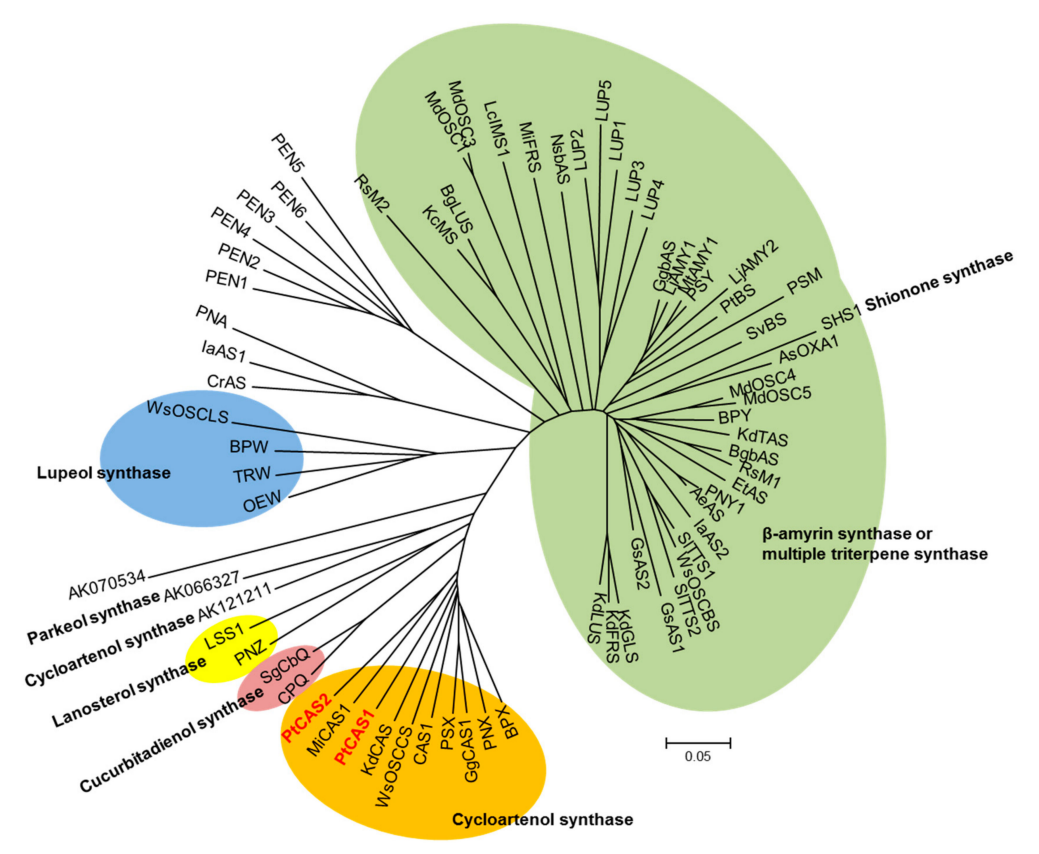

Figure 4. Phylogenetic tree of PtCAS1 and PtCAS2 with other plant OSCs. Gene accession numbers are given in Table S3.

\subsection{Functional Characterization of PtCAS1 and PtCAS2 Genes in Yeast Cells}

We constructed vectors to express the PtCAS1 and PtCAS2 genes by the heterologous expression system in yeast cells. After confirmation of the cDNA sequences, two clones were finally selected and plasmids harboring each gene were used to transform into the yeast mutant GIL77 cells. As per the protocol described by Kushiro et al. [16], transgenic cells were cultured after the addition of galactose for one day. To identify the products in the extracts of the yeast cells expressing PtCAS1 or PtCAS2, we conducted gas chromatography-mass spectrometry (GC-MS) analysis. As shown in Figure 5A, both PtCAS1 and PtCAS2 extracts contained one peak that was not visible with the control cells carrying the empty vector. The two peaks were identified as a cycloartenol by comparing their retention times and mass fragment patterns to the authentic standard (Figure 5B). Therefore, both CAS genes produced cycloartenol by catalyzing 2,3-oxidosqualene.
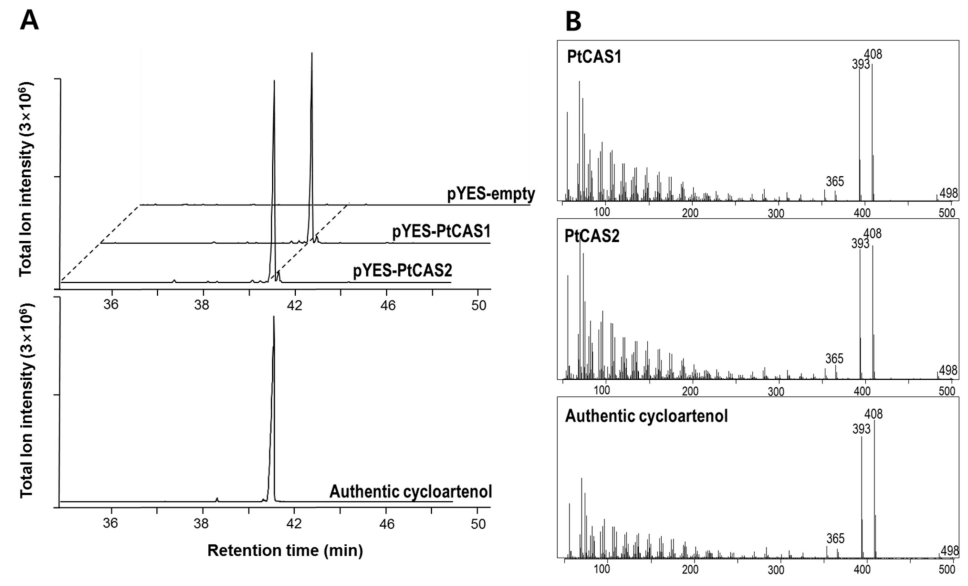

Figure 5. Identification of cycloartenol in yeast cells overexpressing PtCAS1 and PtCAS2 genes by Gas chromatography-mass spectrometry (GC-MS) analysis. (A) GC chromatograms of cycloartenol authentic standard, strain expressing PtCAS1 and PtCAS2 and control yeast strain harboring pYES2 vector; (B) MS spectrum of trimethylsilylated cycloartenol produced from strain expressing PtCAS1 and PtCAS2 and the standard cycloartenol. 


\subsection{Expressions of Two CAS Genes in Organs and in Seedlings Treated with MeJA}

To get insight into the differences in the gene expression of the organs of P. tenuifolia cultured in the field for five months, qRT-PCR analysis was conducted. As shown in Figure 6A, the expression level of the PtCAS1 gene was highest in the roots, whereas the PtCAS2 gene was most abundant in the flowers. These results suggest that the physiological roles may differ between the two genes. The expression of specific genes such as the $b A S$ gene, which leads to up-regulation of triterpenoid saponin biosynthesis, was enhanced in response to MeJA. However, the expression of the CAS genes was not significantly different in spite of MeJA treatment, conceivably, to support phytosterol biosynthesis $[17,18]$. Interestingly, the transcript patterns of the two PtCAS genes of the seedling of P. tenuifolia after MeJA treatment were similar. In the case of the two PtCAS genes of P. tenuifolia, the results of the qRT-PCR analysis showed that the transcripts of PtCAS1 and PtCAS2 genes increased by 1.5- and 2-fold, respectively, in response to MeJA (Figure 6B). This result corresponded to the DEUGs of the PtCAS genes after MeJA treatment. Similarly, Mishra et al. [19] reported that transcript levels of the WsCAS (Withania somnifera cycloartenol synthase) gene of the W. somnifera seedling, which contains a large amount of steroidal triterpene saponins, increased up to 1.65-fold after MeJA treatment. Taken together, these results suggest that the PtCAS genes may contribute to secondary steroidal triterpenoid biosynthesis as well as to phytosterols in P. tenuifolia.
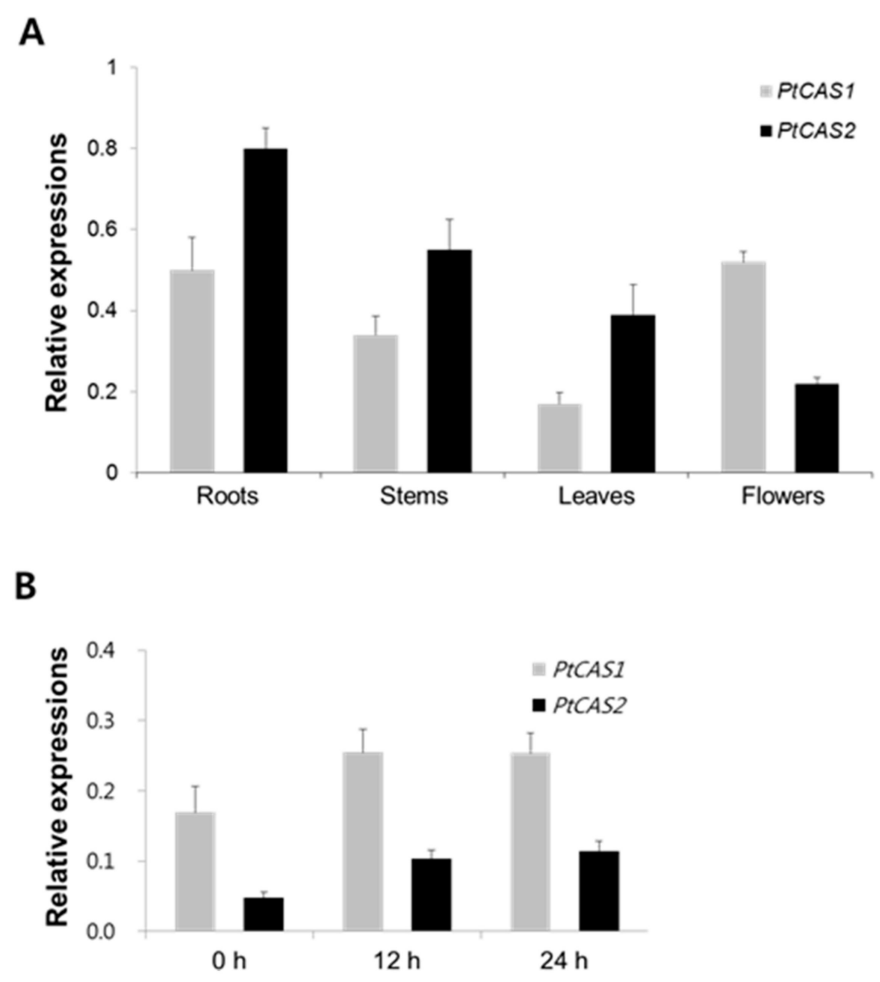

Figure 6. Results of qRT-PCR analysis. (A) Relative gene expression of PtCAS1 and PtCAS2 genes in organs of $P$. tenuifolia cultured in the field for 5 months and (B) time course after MeJA. After pre-cultivation on the $1 / 2$ Murashige and Skoog (MS) medium for 5 weeks, seedlings were elicited by $0.1 \mathrm{mM}$ MeJA. $\beta$-actin gene was used as the reference gene. The error bars represent standard errors from three biological replicates.

\section{Discussion}

In order to uncover novel genes related to sterol and triterpenoid biosynthesis, RNA-seq analysis was conducted of 5-week old seedlings of P. tenuifolia treated with MeJA. In plants, two genes of SQS exist in the A. thaliana and Panax ginseng [20,21]. The gene expression differs by organ and location of the two SQSs. However, only one gene for SQS was identified from the transcriptome dataset. 
By comparing the log fold change (LFC) from the DEUGs of the two samples, the value for TBIU012384 was determined to be 3.36, indicating that this gene is highly expressed in response to MeJA. This result was similar with that of several reported studies, where the SQS gene was upregulated in cells or tissues upon induction with MeJA [21-23]. Five unigenes were annotated as SQE, and the LFC of the TBIU001990 unigene was determined to be the highest. Collectively, given the difference of LFC values observed following MeJA treatment, these results point to the TBIU012384 unigene of SQS and the TBIU001990 unigene of SQE as strong candidiates, which may play a role in controlling the biosynthesis of triterpenoid saponins.

CAS produces cycloartenol as a key precursor, which plays an important role in controlling the biosynthesis of phytosterol via a series of reactions that includes desaturation, hydroxylation, demethylation, etc. CAS genes of several plants have been characterized by heterologous expression in yeast cells $[5,24,25]$. In this work, the results of GC-MS analysis demonstrated that PtCAS1 and PtCAS2 yielded a single cycloartenol product (Figure 5). In contrast to the CAS gene of Arabidopsis, P. tenuifolia contains two genes for CAS in the genome. Thus far, it is not clear why some plants contain multiple copies of CAS gene. According to Zhang et al. [5] one of the genes may exist to safeguard the phytosterol biosynthetic pathway against any fatal mutations that may afflict the other gene. Another theory for the existence of two CAS genes in a plant is that one gene is there to constantly maintain phytosterol production while the other is specifically expressed in response to different environmental conditions. qRT-PCR analysis demonstrated that expressions between the two genes differed in flowers (Figure 6A). It is likely that the PtCAS1 gene is constantly expressed to produce phytosterols and the PtCAS2 gene is responsible for the biosynthesis of steroidal compounds to protect themselves against changing or harsh environments such as pathogens coming into contact with flowers. In addition, CAS is activated to produce steroidal triterpenoids as secondary metabolites, like withanolide [19]. After $12 \mathrm{~h}$ of MeJA treatment on the seedlings of P. tenuifolia, the transcripts of the two CAS genes were higher than that of the control (Figure 6B). This result might indicate that the $C A S$ genes of $P$. tenuifolia also contribute to the biosynthesis of steroidal triterpenoids, although we note that steroidal triterpenoids have not yet been identified.

\section{Materials and Methods}

\subsection{Plant Materials and RNA Extraction}

Seeds of P. tenuifolia were cultured on $1 / 2$ Murashige and Skoog (MS) medium supplemented with $3 \%$ sucrose and $0.8 \%$ agar. Five-week old seedlings were cultured on $1 / 2$ MS liquid medium containing 0.1 mM MeJA for one day, and then total RNA was isolated using the RNeasy Plant kit (Qiagen, Hilden, Germany). The quality of total RNA was measured using the Agilent 2100 Bioanalyzer (Agilent Technologies, Santa Clara, CA, USA).

\subsection{RNA Library Preparation and Sequencing}

RNA-Seq libraries were prepared using a TruSeq RNA Sample Prep Kit according to the manufacturer's manual (Illumina, Inc., San Diego, CA, USA). The poly-A containing mRNA molecules were purified from $1 \mu \mathrm{g}$ of total RNA from each sample using poly-T oligo-attached magnetic beads. The mRNA was fragmented into approximately $200 \mathrm{bp}$ inserts by sonication. The first-strand cDNA of mRNA fragments was synthesized using reverse transcriptase and random hexamer primers, and the second-strand cDNA was then synthesized using DNA Polymerase I and RNase H. The cDNA fragment then underwent an end processing step, which included the addition of a single " $\mathrm{A}$ " base, and then ligation of adapters. The products were then purified and enriched by PCR to amplify the DNA in the library. Size and quantity were confirmed using the Agilent 2200 Tapestation system with High Sensitivity D1000 Tape. After qPCR validation using the KAPA library quantification kit (KAPA Biosystems, Cape Town, South Africa), the libraries were subjected to paired-end sequencing 
with a $100 \mathrm{bp}$ read length using the Illumina HiSeq 2500 platform, yielding on average 74.2 million reads per library.

\subsection{De Novo Assembly and Sequence Annotation}

High quality reads were obtained by removing adaptor, low-quality sequences, and those that contained a high content of unknown bases (N's). De novo transcriptome assembly was performed using Trinity [26], an RNA-seq assembler based on the use of a de Bruijin graph. After assembly, we used the TGI Clustering Tool (TGICL) (Available online: http:/ /www.tigr.org/tdb/tgi/software/) to obtain distinct sequences. The resulting assembled transcripts, known as "unigenes", were analyzed by the TransDecoder tool to predict candidate coding regions within the transcript sequences (Available online: https://transdecoder.github.io/). The unigenes were subjected to searches against the NCBI protein NR (Available online: ftp:/ /ftp.ncbi.nlm.nih.gov/blast/db /), Arabidopsis thaliana TAIR10 (Available online: https:/ / phytozome.jgi.doe.gov/), and Clusters of Orthologous Groups (COG, Available online: http://www.ncbi.nlm.nih.gov/COG/) databases, in addition to BLASTX (Available online: http://blast.ncbi.nlm.nih.gov), employing a cutoff $e$-value of $1.0 \times 10^{-5}$. Estimation of transcript abundance was performed using the RSEM package (Available online: http:// deweylab.github.io/RSEM/), and the number of reads for each unigene was transformed into FPKM (fragments per kilobase million). Based on the quantification results, the differential expression between the samples was analyzed using the R package [27], with a statistical significance of $p<0.01$ and $\geq 1.5$-fold change.

\subsection{Phylogenetic Tree Analysis}

The protein sequences were aligned with the CLUSTALW program and the phylogenetic tree was constructed using the MEGA6 software [28] based on the Neighbor-joining method and bootstrapping for 1000 replicates. Accession numbers of genes used in this analysis are listed in Table S3.

\subsection{Cloning of PtCAS1 and PtCAS2}

In order to obtain the full-length cDNA corresponding to the two PtCAS genes under the same PCR condition reported by Jin et al. [15], forward and reverse primers were designed as described in Table S4. The fragments were separated by agarose gel electrophoresis, purified using a Wizard PCR Preps Kit (Promega, Madison, WI, USA), and then subcloned into the pYES2.1 TOPO vector (Thermo Fisher Scientific, Waltham, MA, USA). Sequences for the PtCAS1 and PtCAS2 genes have been deposited into the GenBank database with accession numbers EU275203 and EU275205, respectively.

\subsection{Heterologous Expression of PtCAS1 and PtCAS2 Genes in Yeast Mutant}

After confirmation of the cDNA sequences of pYES-PtCAS1 and pYES-PtCAS2, the plasmids, including pYES2 (as control), were used for yeast transformation. The transformants were cultured in $10 \mathrm{~mL}$ synthetic complete medium without uracil, containing $20 \mathrm{mg} / \mathrm{L}$ ergosterol, $13 \mathrm{mg} / \mathrm{L}$ hemin, and $5 \mathrm{~g} / \mathrm{L}$ Tween 80 , at $30^{\circ} \mathrm{C}$ and $220 \mathrm{rpm}$. After induction with $2 \%$ galactose, the cells were collected and re-suspended in the same volume of $0.1 \mathrm{M}$ potassium phosphate buffer with the same supplements except ergosterol and Tween 80 , and incubated for one day at $30^{\circ} \mathrm{C}$. Then, the cells from two flasks were collected into one tube, refluxed with $5 \mathrm{~mL} 20 \% \mathrm{KOH}$ and $50 \% \mathrm{EtOH}$, and extracted three times with the same volume of $n$-hexane. The extracts were concentrated under a stream of nitrogen gas and re-suspended in $400 \mu \mathrm{L}$ of $\mathrm{CHCl}_{3}$. Lastly, a $100 \mu \mathrm{L}$-aliquot was re-concentrated and the trimethylsilylated into pyridine with $50 \mu \mathrm{L}$ of $\mathrm{N}, \mathrm{O}$-Bis(trimethylsilyl)trifluoroacetamide for $30 \mathrm{~min}$ at $70{ }^{\circ} \mathrm{C}$.

\subsection{Identification of Products from Two CASs}

GC-MS analysis was performed under the same conditions as described by Wang et al. [29]. A $1 \mu \mathrm{L}$ aliquot of the trimethylsilylated solution was analyzed by a $7890 \mathrm{~N}$ gas chromatography (Agilent 
Technologies) equipped with a mass spectrometric detector (5973 inert MS, Agilent Technologies) and an HP-5 capillary column (Agilent Technologies; length $30 \mathrm{~m}$, i.d. $250 \mu \mathrm{m}, 0.25 \mu \mathrm{m}$, film thickness). Injection condition was at $50^{\circ} \mathrm{C}$ for $2 \mathrm{~min}$. The column temperature program was as follows: $40^{\circ} \mathrm{C} / \mathrm{min}$ ramp to $200{ }^{\circ} \mathrm{C}$, held at $200{ }^{\circ} \mathrm{C}$ for $2 \mathrm{~min}$, followed by an increase to $320^{\circ} \mathrm{C}$ at a rate of $3{ }^{\circ} \mathrm{C} / \mathrm{min}$, and held at $320^{\circ} \mathrm{C}$ for $30 \mathrm{~min}$. Cycloartenol was identified by comparison with the standard (purchased from Chromadex, Irvine, CA, USA).

\subsection{Real-Time RT-PCR Analysis}

Total RNA was extracted from the tissue of one-year-old P. grandiflorum garden plants. First-strand cDNA was synthesized using the AMV (Avian Myeloblastosis Virus) reverse transcriptase (Promega) and $2 \mu \mathrm{g}$ of total RNA. SYBR Green Master Mix (Bio-Rad, Hercules, CA, USA) was used for quantification with the CFX96TM Real-Time System (Bio-Rad) and gene-specific primers (Table S4). PCR conditions were as follows: initial denaturation at $95{ }^{\circ} \mathrm{C}$ for $3 \mathrm{~min}$, followed by 40 cycles of amplification for $15 \mathrm{~s}$ at $95^{\circ} \mathrm{C}$, for $15 \mathrm{~s}$ at $58{ }^{\circ} \mathrm{C}$, and for $30 \mathrm{~s}$ at $72{ }^{\circ} \mathrm{C}$. After completing the reactions, the threshold cycle $\left(C_{t}\right)$ value for each reaction was obtained, and the differences were calculated using the $\Delta \Delta C_{t}$ method, and the $\beta$-actin gene (GenBank accession No. JK517508) was used as an internal control. The fold change in transcript levels for each gene (considered for qRT-PCR) is presented as the mean and standard error of three independent experiments.

\section{Conclusions}

We generated transcriptome data from 5-week-old seedling treated with MeJA for one day by sequencing on Illumina platform. In the data, a total of 11 unigenes related to the OSC gene were detected by gene annotation. Based on sequencing data of two putative CAS unigenes, full-length cDNAs of PtCAS1 and PtCAS2 genes were isolated and analyzed by a heterologous expression system in yeast cells. Results of GC-MS analysis indicated that two PtCAS genes produced cycloartenol as a sole product. However, roles of the two PtCAS genes in relation to phytosterol and steroidal triterpenoid biosynthesis remain unclear, despite having elucidated different expression patterns of each in different tissues. Thus, it will be necessary to further evaluate and differentiate functions of the genes in relation to their roles in controlling phytosterol biosynthesis, by overexpression or silencing them. Results may provide us with a greater understanding of the regulatory mechanisms underlying phytosterol biosynthesis and physiological functions of the two CAS genes of this plant.

Supplementary Materials: Supplementary materials can be found at http:/ / www.mdpi.com/1422-0067/18/11/ 2426/s1.

Acknowledgments: This work was supported by the "Cooperative Research Program for Agricultural Science \& Technology Development (Project No.PJ0023701)" Rural Development Administration, Korea.

Author Contributions: Mei Lan Jin performed the GC-MS analysis and qRT-PCR analysis; Woo Moon Lee performed the transcriptomic analysis and Ok Tae Kim planned this study and wrote this manuscript.

Conflicts of Interest: The authors declare no conflict of interest.

$\begin{array}{ll}\text { Abbreviations } \\ \text { DEUG } & \text { Differential Expression of Unigenes } \\ \text { LFC } & \text { Log Fold Change } \\ \text { FPKM } & \text { Fragments Per Kilobase Million } \\ \text { OSC } & \text { Oxidosqualene Cyclase } \\ \text { MeJA } & \text { Methyl Jasmonate } \\ \text { CAS } & \text { Cycloartenol Synthase } \\ \text { bAS } & \beta \text {-Amyrin Synthase }\end{array}$




\section{References}

1. Phillips, D.R.; Rasbery, J.M.; Bartel, B.; Matsuda, S.P.T. Biosynthetic diversity in plant triterpene cyclization. Curr. Opin. Plant Biol. 2006, 9, 305-314. [CrossRef] [PubMed]

2. Thimmappa, R.; Geisler, K.; Louveau, T.; O'Maille, P.; Osbourn, A. Triterpene biosynthesis in plants. Annu. Rev. Plant Biol. 2014, 65, 225-257. [CrossRef] [PubMed]

3. Babiychuk, E.; Bouvier-Navé, P.; Compagnon, V.; Suzuki, M.; Muranaka, T.; van Montagu, M.; Kushnir, S.; Schaller, H. Allelic mutant series reveal distinct functions for Arabidopsis cycloartenol synthase 1 in cell viability and plastid biogenesis. Proc. Natl. Acad. Sci. USA 2008, 105, 3163-3168. [CrossRef] [PubMed]

4. Husselstein-Muller, T.; Schaller, H.; Benveniste, P. Molecular cloning and expression in yeast of 2,3-oxidosqualene-triterpenoid cyclases from Arabidopsis thaliana. Plant Mol. Biol. 2001, 45, 75-92. [CrossRef] [PubMed]

5. Zhang, H.; Shibuya, M.; Yokota, S.; Ebizuka, Y. Oxidosqualene cyclases from cell suspension cultures of Betula platyphylla var. japonica: Molecular evolution of oxidosqualene cyclases in higher plants. Biol. Pharm. Bull. 2003, 26, 642-650. [CrossRef] [PubMed]

6. Liu, J.; Yang, X.; He, J.; Xia, M.; Xu, L.; Yang, S. Structure analysis of triterpenesaponins in Polygala tenuifolia by electrospray ionization ion trap multiple-stage mass spectrometry. J. Mass Spectrometry 2007, 42, 861-873. [CrossRef] [PubMed]

7. Klein, L.C., Jr.; de Andrade, S.F.; Cechinel Filho, V. A pharmacognostic approach to the Polygala genus: Phytochemical and pharmacological aspects. Chem. Biodivers. 2012, 9, 181-209. [CrossRef] [PubMed]

8. Ramirez-Estrada, K.; Vidal-Limon, H.; Hidalgo, D.; Moyano, E.; Golenioswki, M.; Cusidó, R.M.; Palazon, J. Elicitation, an effective strategy for the biotechnological production of bioactive high-added value compounds in plant cell factories. Molecules 2016, 21, 182. [CrossRef] [PubMed]

9. Santino, A.; Taurino, M.; de Domenico, S.; Bonsegna, S.; Poltronieri, P.; Pastor, V.; Flors, V. Jasmonate signaling in plant development and defense response to multiple (a)biotic stresses. Plant Cell Rep. 2013, 32, 1085-1098. [CrossRef] [PubMed]

10. Tian, H.; Xu, X.; Zhang, F.; Wang, Y.; Guo, S.; Qin, X.; Du, G. Analysis of Polygala tenuifolia transcriptome and description of secondary metabolite biosynthetic pathways by Illumina sequencing. Int. J. Genom. 2015, 2015, 782635.

11. Jensen, L.J.; Julien, P.; Kuhn, M.; von Mering, C.; Muller, J.; Doerks, T.; Bork, P. eggNOG: Automated construction and annotation of orthologous groups of genes. Nucleic Acids Res. 2008, 36, D250-D254. [CrossRef] [PubMed]

12. Monden, Y.; Tahara, M. Genetic linkage analysis using DNA markers in sweetpotato. Breed. Sci. 2017, 67, 41-51. [CrossRef] [PubMed]

13. Ismail, N.A.; Rafii, M.Y.; Mahmud, T.M.; Hanafi, M.M.; Miah, G. Molecular markers: A potential resource for ginger genetic diversity studies. Mol. Biol. Rep. 2016, 43, 1347-1358. [CrossRef] [PubMed]

14. Rasbery, J.M.; Shan, H.; LeClair, R.J.; Norman, M.; Matsuda, SPT.; Bartel, B. Arabidopsis thaliana squalene epoxidase 1 is essential for root and seed development. J. Biol. Chem. 2007, 282, 17002-17013. [CrossRef] [PubMed]

15. Jin, M.L.; Lee, D.Y.; Um, Y.; Lee, J.H.; Park, C.G.; Jetter, R.; Kim, O.T. Isolation and characterization of an oxidosqualene cyclase gene encoding a $\beta$-amyrin synthase involved in Polygala tenuifolia Willd. saponin biosynthesis. Plant Cell Rep. 2014, 33, 511-519. [CrossRef] [PubMed]

16. Kushiro, T.; Shibuya, M.; Ebizuka, Y. $\beta$-amyrin synthase-Cloning of oxidosqualene cyclase that catalyzes the formation of the most popular triterpene among higher plants. Eur. J. Biochem. 1998, 256, 238-244. [CrossRef] [PubMed]

17. Hayashi, H.; Huang, P.Y.; Inoue, K. Up-regulation of soyasaponin biosynthesis by methyl jasmonate in cultured cells of Glycyrrhiza glabra. Plant Cell Physiol. 2003, 44, 404-411. [CrossRef] [PubMed]

18. Suzuki, H.; Achnine, L.; Xu, R.; Matsuda, SPT.; Dixon, R.A. A genomic approach to the early stages of triterpene saponin biosynthesis in Medicago truncatula. Plant J. 2002, 32, 1033-1048. [CrossRef] [PubMed]

19. Mishra, S.; Bansal, S.; Mishra, B.; Sangwan, R.S.; Asha Jadaun, J.S.; Sangwan, N.S. RNAi and homologous over-expression based functional approaches reveal triterpenoid synthase gene-cycloartenol synthase is involved in downstream withanolide biosynthesis in Withania somnifera. PLoS ONE 2016, 11, e0149691. [CrossRef] [PubMed] 
20. Kribii, R.; Arró, M.; Del Arco, A.; González, V.; Balcells, L.; Delourme, D.; Ferrer, A.; Karst, F.; Boronat, A. Cloning and characterization of the Arabidopsis thaliana SQS1 gene encoding squalene synthase-Involvement of the C-terminal region of the enzyme in the channeling of squalene through the sterol pathway. Eur. J. Biochem. 1997, 249, 61-69. [CrossRef] [PubMed]

21. Kim, T.D.; Han, J.Y.; Huh, G.H.; Choi, Y.E. Expression and functional characterization of three squalene synthase genes associated with saponin biosynthesis in Panax ginseng. Plant Cell Physiol. 2011, 52, 125-137. [CrossRef] [PubMed]

22. Kim, O.T.; Kim, M.Y.; Huh, S.M.; Bai, D.G.; Ahn, J.C.; Hwang, B. Cloning of a cDNA probably encoding oxidosqualene cyclase associated with asiaticoside biosynthesis from Centella asiatica (L.) Urban. Plant Cell Rep. 2005, 24, 304-311. [CrossRef] [PubMed]

23. Kim, Y.S.; Cho, J.H.; Park, S.; Han, J.Y.; Back, K.; Choi, Y.E. Gene regulation patterns in triterpene biosynthetic pathway driven by overexpression of squalene synthase and methyl jasmonate elicitation in Bupleurum falcatum. Planta 2011, 233, 343-355. [CrossRef] [PubMed]

24. Dhar, N.; Rana, S.; Razdan, S.; Bhat, W.W.; Hussain, A.; Dhar, R.S.; Vaishnavi, S.; Hamid, A.; Vishwakarma, R.; Lattoo, S.K. Cloning and functional characterization of three branch point oxidosqualene cyclases from Withania somnifera (L.) dunal. J. Biol. Chem. 2014, 289, 17249-17267. [CrossRef] [PubMed]

25. Kawano, N.; Ichinose, K.; Ebizuka, Y. Molecular cloning and functional expression of cDNAs encoding oxidosqualene cyclases from Costus speciosus. Biol. Pharm. Bull. 2003, 25, 477-482. [CrossRef]

26. Grabherr, M.G.; Haas, B.J.; Yassour, M.; Levin, J.Z.; Thompson, D.A.; Amit, I.; Adiconis, X.; Fan, L.; Raychowdhury, R.; Zeng, Q.; et al. Full-length transcriptome assembly from RNA-seq data without a reference genome. Nat. Biotechnol. 2011, 29, 644-652. [CrossRef] [PubMed]

27. Sun, J.; Nishiyama, T.; Shimizu, K.; Kadota, K. TCC: An R package for comparing tag count data with robust normalization strategies. BMC Bioinform. 2013, 14, 219. [CrossRef] [PubMed]

28. Tamura, K.; Stecher, G.; Peterson, D.; Filipski, A.; Kumar, S. MEGA6: Molecular evolutionary genetics analysis version 6.0. Mol. Biol. Evol. 2013, 30, 2725-2729. [CrossRef] [PubMed]

29. Wang, Z.; Yeats, T.; Han, H.; Jetter, R. Cloning and characterization of oxidosqualene cyclases from Kalanchoe daigremontiana: Enzymes catalyzing up to 10 rearrangement steps yielding friedelin and other triterpenoids. J. Biol. Chem. 2010, 285, 29703-29712. [CrossRef] [PubMed] 